\title{
Latour, Bruno: Das terrestrische Manifest, übers. v. Bernd Schwibs, 136 S., Suhrkamp, Berlin 2018 (frz. 2017).
}

\section{Felix Heidenreich}

Online publiziert: 18. Mai 2020

(C) Der/die Autor(en) 2020

Die Versuchung, die Wahl Donald Trumps als Ergebnis einer zufälligen Konstellation zu charakterisieren, in der die Fernsehprominenz des einen, die Unbeliebtheit der anderen, der externe Einfluss durch Russland und die innere Spaltung der Demokraten zusammenkamen, ist groß. Ganz anders geht Bruno Latour mit Trumps Wahl um: Er versucht in seinem Essay nichts weniger als eine epochale Neuordnung des politischen Feldes, die Trump als einen Indikator für Grundlegendes nimmt. Latour entwickelt auf den rund 110 Seiten eine teils essayistisch leichte, teils konzeptionell dichte Beschreibung einer neuen Gesamtlage. Das im Titel angekündigte neue Ziel progressiven politischen Handelns, das ,TERRESTRISCHE“, will Latour gerne in Majuskeln geschrieben wissen, um den begrifflichen Status dieser Formulierung zu markieren.

Im akademischen Betrieb mag ein solcher Essay zunächst rätselhaft, äußerst spekulativ, ja schlicht seltsam anmuten. Die Ausrufung neuer Weltalter gehört zu den festen Topoi der Philosophiegeschichte, die man heute analysieren, aber nicht reproduzieren sollte. Doch der Autor entwickelt die Grundthese in kleinen und nachvollziehbaren Schritten. Die Argumentation beginnt mit einer scharfen These: Das Phänomen Donald Trump müsse als epochales Ereignis betrachtet werden. Mit Trump und dem Trumpismus betritt, so Latour, eine Denkhaltung die Bühne, die wir so noch nicht gesehen haben. Was genau ist so neu am Trumpismus? Er verbindet den Anspruch auf grenzenlosen Konsum mit der kognitiven und nationalen Totalbegrenzung. Damit kombiniert Trump zwei sich eigentlich widersprechende Weltsichten: Wachstumsglaube und Unbeschränktheitskult einerseits und eine Haltung der Realitätsexklusion andererseits. Konsum und Verschwendung sollen unbegrenzt weitergehen, während man eine Mauer baut und auch die Realität des Klimawandels ausschließen will.

Diese Einordnung verschiebt zunächst den Fokus in der Analyse des Trumpismus. Die Leugnung des Klimawandels (und seiner Ursachen) ist für diese Weltsicht nicht etwa akzidentiell, sondern essenziell: Müssten die Trumpisten einräumen, dass es einen Klimawandel gibt und dass er vom Menschen gemacht wird, würde ihr Begriff einer unbeschränkt als Konsumobjekt zur Verfügung stehenden Natur in sich zusam-

F. Heidenreich $(\bowtie)$

Universität Stuttgart, Stuttgart, Deutschland

E-Mail: felix.heidenreich@izkt.uni-stuttgart.de 
menbrechen. Die Genialität Trumps besteht folglich darin, so Latour, dass der kognitive Provinzialismus reaktionären Denkens einerseits und die Aufbruchsrhetorik des Globalismus andererseits ineinandergeschraubt werden: Wer Trump wählt, will die Welt mit einer Mauer aussperren und sie dennoch unbegrenzt verkonsumieren dürfen: „Drill, Baby, drill!“ Was einst progressiv hieß - Wachstum, Globalisierung, Liberalisierung - verbündet sich hier auf verquere Weise mit Tribalismus, Rassismus, identitärem Denken. Trump hat damit ein Wolkenkuckucksheim erfunden, dass bisher so noch niemand zu formulieren wagte: Der Anspruch des Säuglings, konsumieren zu dürfen, ohne Verantwortung tragen zu müssen. Latour zeichnet damit das Psychogramm einer kollektiven Regression: Die Welt wird gegessen, ohne zur Kenntnis genommen zu werden.

Damit hat Trump ein Ziel formuliert, dass einerseits den klassischen Konservativismus und andererseits den klassischen Fortschrittsglauben ersetzt: Trump ist weder links noch rechts, weder vorne noch hinten, sondern einfach ,daneben', jenseits des Realitätsbezugs. Diese Umbesetzung muss man, so Latours ernüchternde Forderung, zunächst als ideengeschichtliches Ereignis ernst nehmen. Nur so lässt sich angemessen antworten.

Latours Gegenentwurf ergibt sich als symmetrische Spiegelung. Der Pseudologia phantastica des Trump setzt Latour einen neuen „Attraktor“ entgegen, den er „das Terrestrische“ nennt. Spiegelt man Trumps Ideologie, so erhält man einen ethischen, menschenrechtlich fundierten Universalismus einerseits mit einer ökologisch begründeten Selbstbeschränkung auf den Nahraum andererseits. Aber ,das Terrestrische" soll mehr sein als global denken und lokal handeln. Der Konnotationsraum der französischen terre ist nur schwer ins Deutsche zu übertragen. Es geht hier gerade nicht um Boden wie bei ,Blut und Boden', sondern um jenen Boden, den beispielsweise Weinbauern pflegen und vererben. Terrestrisch denken und handeln heißt dann so etwas wie: sich auf einen Nahraum beschränken, sich für eine Biosphäre verantwortlich fühlen, irgendwo eine (durchaus gemachte, entworfene) ,Heimat ' finden. Mit identitärem Denken und Heimattümelei hat dies gerade nichts zu tun; eher noch mit der Einsicht, dass der Planet noch mehr Massentourismus, noch mehr Mobilität, noch mehr „Bodenlosigkeit“ nicht vertragen kann. Der Übersetzer der deutschen Ausgabe Bernd Schwibs hat sehr sorgfältig daran gearbeitet, auch im Deutschen die richtige Tonlage zu treffen.

In den Schlussabschnitten wendet Latour seine Antwort auf den Trumpismus europapolitisch. Wer ,terrestrisch“ denkt, ,landet“ irgendwo: „Landen heißt zwangsläufig, an einer bestimmten Stelle zu landen. Das Folgende soll als Eröffnung zu einer höchst riskanten diplomatischen Unterredung mit denjenigen verstanden werden, mit denen man zusammen leben will. Nun, ich möchte in Europa landen!“ (S. 115). Ein vollständig konträr zum Trumpismus gedachtes Europa wird so zur neuen Zielgröße erklärt.

Latours 2017 in Originalsprache erschienener Text ist von einem gewissen Optimismus getragen. Würde der Autor auch heute noch eine solche Einladung aussprechen? Wie geht man mit jenen um, die alle Diplomatie mit Füßen treten, mit denen man aber zusammen leben muss? Könnte es sein, dass die von Latour beschriebene Neuordnung des politischen Feldes viel mehr Eskalationspotenzial birgt, als es der Autor 2017 andeutete? Dass sich mit dem Brexit-Votum und Trumps Wahl grund- 
legende Dinge verschoben haben, spürt man intuitiv. Latours Essay regt dazu an, nicht nur die Fremd-, sondern auch die Selbstbestimmung genauer zu durchdenken.

Funding Open Access funding provided by Projekt DEAL.

Open Access Dieser Artikel wird unter der Creative Commons Namensnennung 4.0 International Lizenz veröffentlicht, welche die Nutzung, Vervielfältigung, Bearbeitung, Verbreitung und Wiedergabe in jeglichem Medium und Format erlaubt, sofern Sie den/die ursprünglichen Autor(en) und die Quelle ordnungsgemäß nennen, einen Link zur Creative Commons Lizenz beifügen und angeben, ob Änderungen vorgenommen wurden.

Die in diesem Artikel enthaltenen Bilder und sonstiges Drittmaterial unterliegen ebenfalls der genannten Creative Commons Lizenz, sofern sich aus der Abbildungslegende nichts anderes ergibt. Sofern das betreffende Material nicht unter der genannten Creative Commons Lizenz steht und die betreffende Handlung nicht nach gesetzlichen Vorschriften erlaubt ist, ist für die oben aufgeführten Weiterverwendungen des Materials die Einwilligung des jeweiligen Rechteinhabers einzuholen.

Weitere Details zur Lizenz entnehmen Sie bitte der Lizenzinformation auf http://creativecommons.org/ licenses/by/4.0/deed.de.

\title{
Biebricher, Thomas: Geistig-moralische Wende. Die Erschöpfung des deutschen Konservatismus, 320 S., Matthes \& Seitz, Berlin 2018.
}

\author{
Jens Hacke \\ Online publiziert: 15. Juni 2020 \\ (C) Der/die Autor(en) 2020
}

Thomas Biebrichers Bestandsaufnahme des deutschen Konservatismus hat verdientermaßen ein sehr positives Presseecho gefunden. Es handelt sich um eine gedankenreiche Studie, die höchst lesbar geschrieben ist und die Politikwissenschaft daran erinnert, dass sie in der Lage sein sollte, ihre Befunde einem interessierten Publikum verständlich mitzuteilen. Biebrichers Essay zur Lage der Unionsparteien, die er im Wesentlichen als politische Repräsentation des Konservatismus begreift, erschien in bewegter Zeit, als der Wahlkampf um den Parteivorsitz und die Nachfolge Angela Merkels in vollem Gange war. Die aktuellen Bezüge zur Krise der Union, die eine Reihe von Wahlniederlagen erfahren hatte und die sich seither auf der Su-

\footnotetext{
J. Hacke $(\square)$

Humboldt-Universität zu Berlin, Berlin, Deutschland

E-Mail: jens.hacke@sowi.hu-berlin.de
} 